\title{
Adaptive Fitts for Adaptive Interface
}

\author{
Sajib Hasan
}

\begin{abstract}
Adaptive interface would enable Human Computer Interaction apply machine learning to cope with human carelessness (mistakes), understand user performance level and provide an interaction interface accordingly. This study tends to translate the theoretical issues of human task into working model by investigating and implementing the predicting equation of human psychomotor behavior to a rapid and aimed movement, developed by Paul Fitt in 1954. The study finds logarithmic speed-accuracy trade-off and predict user performance in a common task "point-select" using common input device mouse. The performance of user is visualized as an evidence and this visualization make a valuable step toward understanding the change required in user interface to make the interface adaptive and consistent. It proposed a method of calculating the amount of change required through learning; add extension to the theory of machine intelligence and increase knowledge of Fitts applicability in terms of machine learning.
\end{abstract}

Keywords--HCI, Fitts' Law, Speed-Accuracy, Machine Learning, Linear Regression.

\section{INTRODUCTION}

When it comes to user interface design in Human Computer Interaction it is reasonable to think how design use innovation and reengineering not just objects, but entire systems with an understanding of how users operate in practice in the task environment [1]. Modeling human motion with acquisition of human psychomotor behavior is a popular research area and has interest in HCI [2].

Much of what people do is information processing. Human sense organs sense and transmit to the brain an enormous amount of information per second as an input [18]. In the study of Human Computer Interaction performance of human motor processor is modelled and predicted using information processing analogy and Fitts law is an appropriate tool for predicting pointing time. Fitts suggested that, motor control to achieve a movement task is information processing.

In this study Fitts' law plays a role of a predictive model of human psychomotor behavior, since it provides an important predicting equation for pointing time to a rapid and aimed movement, developed by Paul Fitt (1954) [3][8]. A movement task's difficulty (ID, the "index of difficulty") for a motor is quantified based on Shannon's logarithmic expression by the metric "bits". The computation can be stated as below-

$$
I D=\log _{2}\left(\frac{2 A}{W}\right)
$$

Sajib Hasan

American International University-Bangladesh

Dhaka, Bangladesh

sajib.hasan@aiub.edu
A being the distance to the target, and $\mathrm{W}$ the width of the target. Since A and $\mathrm{W}$ are both distances, their ratio within the logarithm is without units. The unit "bits" emerges from the somewhat arbitrary choice of base 2 for the logarithm [3][4][6].

According to Fitts' [4], the time to complete a movement task (total movement time -MT) is predicted using a simpler linear equation, where a linear function of the index of difficulty (ID) of pointing a target can be determined by regressing MT on ID. The regression line equation is:

$$
M T=a+b I D,
$$

where $a$ and $b$ are regression coefficients. $b$ is the slope coefficient and $a$ is the intercept coefficient. Intercept coefficient, $a$, is sometimes viewed as an error term. The usual form of Fitts' law expanded as follows:

$$
M T=a+b \log _{2}\left(\frac{2 A}{W}\right)
$$

Throughout the study input device has been investigated on measure case of use, of accuracy and speed of a user. Controlled experiments have done using a group of objects, input devices and task conditions. Tasks are devised to cover a range of difficulties by varying distance and width. For each task condition, multiple trials are conducted and the time to execute each is recorded and stored electronically for statistical analysis. Errors are also recorded to analyzed and discuss. Objects and actions represented on a graphical display are interacted by pointing device.

Main objective of this study is to find logarithmic speedaccuracy trade-off and predict user performance in common task "point-select" using common input device mouse. As mathematical characterization of Fitt's law includes logarithmic function, relationship between size and reaction time gives a curve which indicates that small increases in size for small objects make it much easier to select them. And the same applies for changes in target distance.

Advance objective of this study is to gain individual user performance with small change in the selection interface. To do this the system may need to know many conditions like for example whether this is the first time the user has been in the system. Using the Artificial Intelligence metaphor, the goal of this part of the study is to attribute human characteristics to the interface.

Following this introductory section, the background study section justifies Fitts' model as analogous to the information processing with a brief discussion on information transmission, measurement of information and Shannon information theorem. Rest of the section discuss about the development of 
Fitts model in HCI and retest some of Fitts hypothesis to use them to achieve advance objective of this study.

\section{BACKGROUND STUDY}

\section{A. Interaction Model}

Interaction does not occur within a vacuum. Darko Brodi and Alessia Amelio, 2016 describes nature of interaction in three-level: psycho-social, metal functions and sensory-motor which highlights the work of Clarke in 1986 [23]. The threelevel of Human-Computer-Interface model has been shown in Fig. 1.

1) Psycho-Social (Level 1): At this level the human element is the goal that the user us pursuing. Computer elements are virtual objects which user can manipulate. Computer element exists to support the fulfilment of user goals. Effective communication is established when computer element (virtual object) match user's objectives [4].

2) Mental Functions (Level 2): At this level both elements of psycho-social level is translated. The computer element of this level is represented by the computer programs which enable the user's goal to be accomplished. The human element of this level is the mental mechanisms (memory, perception, cognition) that the user performs to operate the computer program. When a user using computer element (program), the user will have some idea in his mind about the way the program will perform the necessary task. The computer also contains a model of the task [4].

3) Sensory-Motor (Level 3): At this level human effectors and senses (e.g. hands and eyes) and computer Input-Output devices (e.g. mouse) will interact with the physical objects within the system [4].

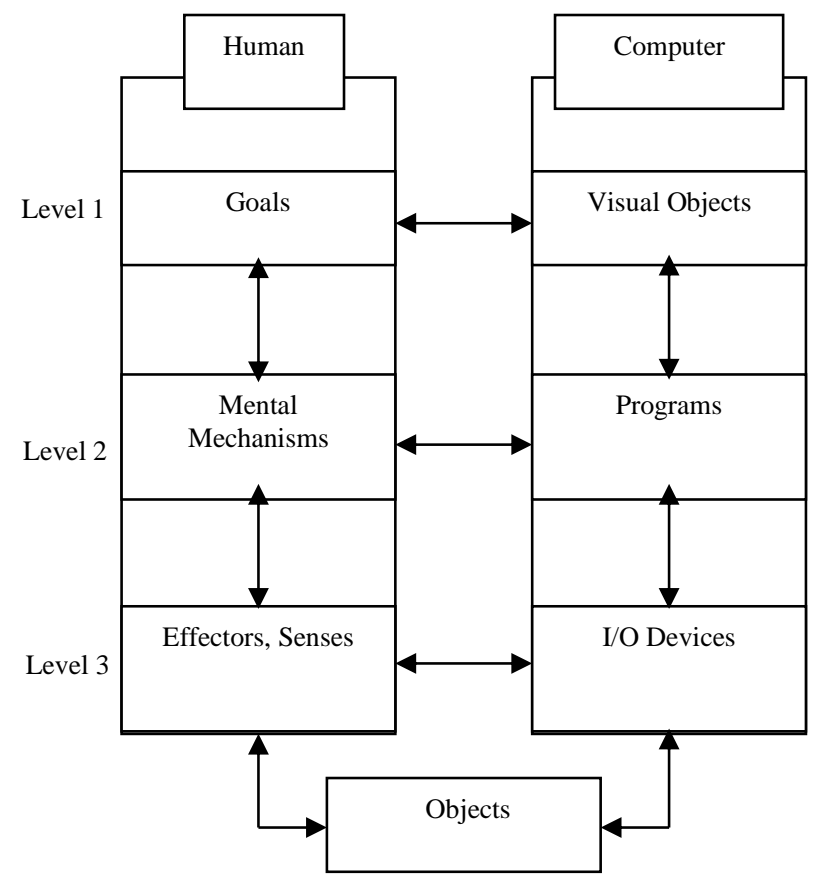

Fig. 1. Three-Level Human-Computer-Interface Model (Andy Smith, 1997, p.201)

\section{B. Information Transmission and Performance Measurement}

Human are transmitter of information and information transmission can be a mean of measuring human performance. The realization of movements in Fitts' model is analogous to the transmission of information in electronic system [14].

1) Information Transmitted: Human response to stimuli thus information is transmitted. Whatever amount of the stimulus information is represented in the subject's response is the amount of information transmitted. When only one response occurs for a given stimulus maximum information is transmitted.

In Fig. 2, $H_{X}$ is Stimulus information, $H_{Y}$ is Response information and $H_{(X, Y)}$ is Cell information [14]. The amount of transmitted information can be calculated using the following equation- $H_{T}=H_{X}+H_{Y}-H_{(X, Y)}$.

2) Performance Measurement: Human performance is measured in terms of stimulus-response relationship. The measurement of how well and fast a subject response to any stimuli is the measurement of subjects' motor performance.

The measurement of motor response in Fitts method is based on the ratio of accuracy to amplitude, and thus resembles the Weber ratio in sensory psychophysics. The WeberFrencher law compare performance. This law relates the degree of response of a sense organ and the intensity of the stimulus. Paul Fitts in 1954 originally proposed the term $\log _{2}\left(\frac{2 A}{W}\right)$ as "index of difficulty (ID)". Many authors proposed different definition of the index of difficulty and perform different experiments. Few are shortly described below-

3) Interactive correction model: Crossman (1956) with his "interactive correction model" suggested that aiming movement is sum of a series of discrete sub-movements thus the ID (index of difficulty) is interpreted as the difference between two fundamental quantities $\log 2$ (W) and $\log 2$ (A). $\log _{2}$ (A) measures the entropy of the endpoint distribution and $\log _{2}(2 \mathrm{~A})$ measures the entropy of a hypothetical initial distribution of motion amplitudes" [19]. The mathematical form of the equation is- $M T=a+b \log _{2}\left(\frac{A}{W}\right)$.

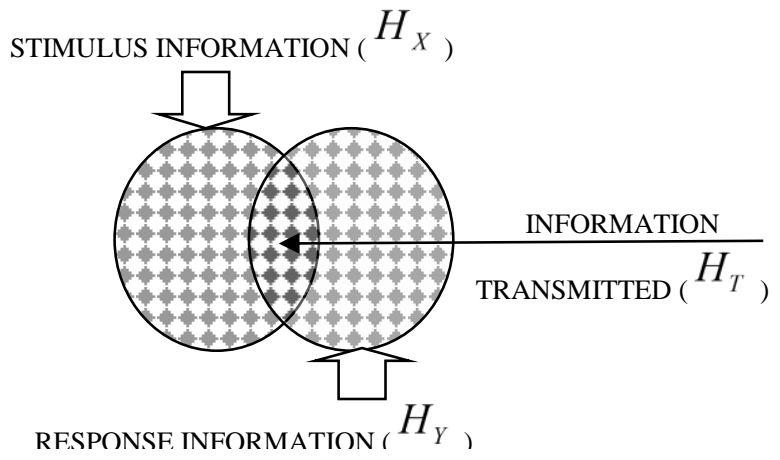


Fig. 2. The Relationship between Stimulus, Response, and Transmitted Information

b) Effective target width: Welford (1968) described the concept of "effective target width". He suggested that subjects do not utilize the entire target area (W) rather they only utilize the nearly half of the target area [19]. Welford modified the equation as- $M T=k \log _{2}\left(\frac{A}{W}+0.5\right)$, $\mathrm{k}$ is an experimentally determined constant. Welford et al. (1969) later advanced the equation where he separately relates movement time with amplitude and with target width. The revised equation is $M T=a+b \operatorname{Alog}_{2}(A)+b_{W} \log _{2}\left(\frac{1}{W}\right)$.

c) Jagacinski index of difficulty: Jagacinski et al (1980b) proposed another alternative index of difficulty as function of the target's speed [19]. Jagacinski conducted experiment on moving targets with constant velocity and incorporates velocity factor as $M T=c+d A+e^{(V+1)\left(\frac{1}{W}-1\right)}$, where $\mathrm{V}$ represents mean velocity of the target movement and $\mathrm{c}, \mathrm{d}$, e represents as fitting constants.

d) Hoffmann Movement Time: Hoffmann (1991a) has proposed different mathematical form of capturing the speed when a target is moving (describing Jagacinski data) [19]. He proposed an equation $M T=-a+b(c+D) \log _{2}\left(\frac{2 A}{W}\right)$, where $D$ is the delay, $a, b$, and $c$ are regression coefficients.

e) MacKenzie Movement Time: MacKenzie (1989; 1992) showed that Fitts' equation slightly deviates from the fundamental theorem 17 of Shannon [19]. He proposed a corrected equation of Fitts as $M T=a+b \log _{2}\left(\frac{A}{W}+1\right)$.

f) Gan and Hoffmann movement time: Gan and Hoffmann (1988) found that for smaller index of difficulty is movement time (MT) strongly dependent on movement amplitude (A) and there are no significant effects from target width [19]. So, he proposed that for less difficult task movement time (MT) can be predicted by an equation relating only $\mathrm{A}$ and stated as $M T=a+b \sqrt{A}$.

g) Johnsgard (1994) movement time: Johnsgard (1994) modified the equation of MacKenzie and included the effect of deice gain $(\mathrm{G})[19]$. His proposed equation is $M T=a\left(\frac{A}{W}\right)^{b}$.

\section{Movement Control and Human Performance}

In general movement is simply the displacement of a limb to a target, followed by a grasping or releasing movement. Some interaction involves series of movements as a sting of individual movements, each directed to a separate target. Evidence indicates that the control of a well-predicted series of movements is quite different than the control of a single, precise movement directed to a target [17].

1) Eye-Hand Coordination: There is relationship between visual input and motor output. Studies have shown that when hand moves rapidly to a target, eyes generally move to the target location shortly before the hand. The delay measured between eye and hand movements typically range from 60 to 100 msec. [9]. Eyes movement to a target support hand movement even when focus is taken away from the target or target cannot be seen. So, it indicates that spatial information (about where the eye is pointing) can be accessed by hand. When visual input of a person indicates that the target will be missed is translated into appropriate corrective movements [17]. Adjustments to small changes in the eye-hand relationship takes few seconds in adults as it follows years of experience with a constant correspondence between input and output [17].

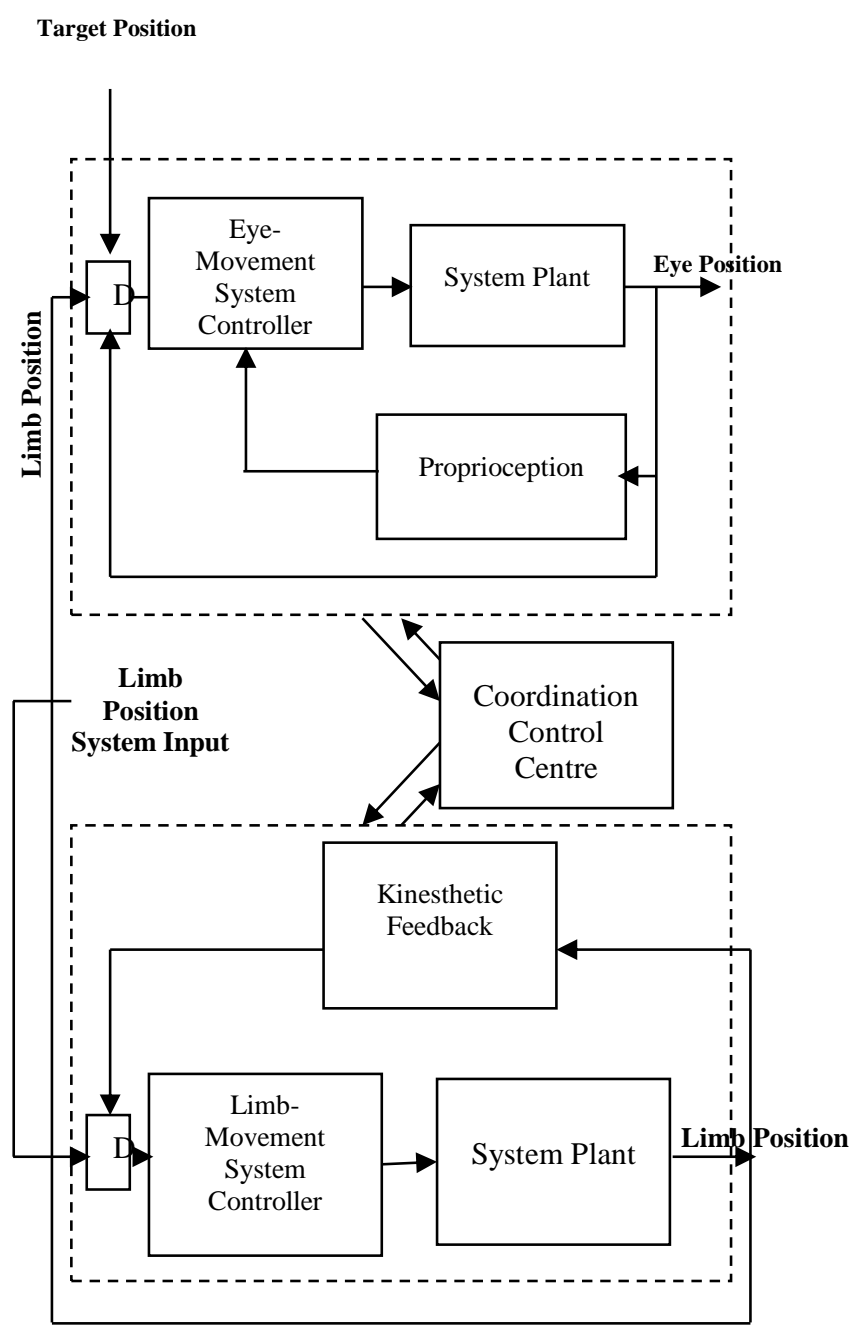

Fig. 3. Control Diagram of Eye-Hand Coordination (David A. Rosenbaum, 1991, p.361)

\section{MeTHODOLOGY}

The main objective of the study was set to model point-andselect task on graphical elements (targets) to predict user performance in mouse based cursor movements using Fitts' paradigm. This part of the study raises few hypotheses to set a direction to facilitate pointing task by changing Fitts two parameter (distance and width of a target). 


\section{A. Setup and Interface Design}

When human interact with computer, physical behavior of the user and the interface perform a task, thus an interface has been designed to focus on user interaction.

1) Participants: 20 volunteers, of different gender and age participated in a target pointing experiment. Like Fitts original experiment, participants did reciprocal pointing on pair of horizontal square shape target with a mouse. Participants are presented with task and are instructed to perform as quickly as possible.

2) Procedure: Subjects performed 27 trials on "pointselect" task. Subject moved the cursor back and forth between the targets and selected each target by pressing and releasing a mouse button. A text "click" appeared just below the target indicates the target to be selected. After each selection the text moved to the opposite target, thereby guiding subjects through the block of trials.

3) Apparatus: Experiments are conducted in a laptop computer (HP) with a screen size 9 Inch. Each pixel of the screen was $0.2645 \mathrm{~mm}$ wide. Although mice are the commonly used pointing device by users, this study used a laptop in the experiment because the data collected from an absolute positioning device corresponds more directly and reliably to physical limb movements, potentially allowing for more kinds of analysis.

4) Task: Task in this study is like traditional Fitts reciprocal tapping task. Task are designed in three levels; each level has three tasks. Target amplitude and target width designed in three levels as well, each level has three different target amplitude and target width combination.

The experiment was sequenced by trials, blocks, and sessions. Each trial was a single point-select task; each block was a series of 3 trials; each session was a series of 3 blocks covering, a systematic order, and the 9 combinations of target amplitude and target width. The total trial can be computed as follows-

20 participants, 9 (A \& W) combinations per block, and 3 trials per A, W combinations gives a total $(20 * 9 * 3)=540$ trials in total

\section{B. Database Design}

User trial data are generated and then recorded in a physical database. Database is created using SQL command. A separate python program is developed to create a database file with several tables. This database program is then implicitly called inside the main program. Trials of users are recorded in a specific format to perform later analysis.

1) User Access: User access is provided with a username and password field. A separate form pops up when user click on the log in submenu. Database can identify new and existing user. Separate experimental phase is designed for new and existing user

2) Data Recording: A table is created with the name 'dis width id' for once to store target width, target distance and task difficulty (index of difficulty) with 27 task indexes.
This table gives a set of different distance and width combination used in the model to generate task difficulty for further analysis of Fitts parameter. User access data and their performance coefficients (slope and intercept) are recorded in the database. A table is created in the name of the individual user (provided during log-in time) to record identical user movement time on 27 tasks they perform. Movement time (MT) and index of performance (IP) is calculated during the task process and recorded in the table (table in the name of individual user). After completing 27 tasks the coefficients for the individual users can be calculated and the 'user' table is updated with calculated slope, intercept and average performance data added to the corresponding fields in the specific row provided for that identical user in the 'user' table with SQL update command.

\section{Problem definition and Hypothesis}

In Fitts law task precision (accuracy) is quantified by index of difficulty (ID) and specified by target parameters (distance and width). Speed is represented by the time (Movement Time) to complete a task. Below are the hypotheses tested and discussed in the result and analysis part of this chapter to consider techniques of improving human pointing performance using mouse, thus gain advance objective of the study in the next part.

Hypothesis 1: Movement Time (MT) increases linearly with the Index of Difficulty (ID).

Hypothesis 2: Transmitting ID bits requires $\mathrm{a}+\mathrm{b}$ *ID seconds in total.

Hypothesis 3: A target that is twice as far away and twice as large requires the same time to acquire.

\section{RESULTS AND DISCUSSION}

Recorded data in the database are retrieved to perform statistical analysis. MatLab tool are used inside python program to do matrix operation and regression analysis of the data.

\section{A. Results}

Results are analyzed to gain the techniques of designing graphical user interface for the improvement of human pointing performance using mouse.

1) ID vs. MT: After trials of 20 subjects their movement times have been recorded with corresponding index of difficulty. A linear regression analysis has been conducted based on the data where ID was an independent variable and MT was a dependent variable. The predictive model of this study joins all 20 users' trial data to regress MT on ID to find

model coefficients (slope and intercept) and draw model regression line on a scatter plot of ID vs. MT. The model can also find individual user coefficients (user slope and intercept) and draw that user regression line in the same scatter plot giving a possible visible comparison between them (model coefficients and user coefficients), which is shown in Fig. 4. 
2) Correlation Coefficients ( $b, a)$ and Movement Time (MT): After completing 27 trials by everyone, user correlation coefficient (r), slope (b) and intercept (a) of the user data is calculated. Three user trial data has been examined in an excel file to find out if the prediction equation 2 (MT=a $+b^{*}$ ID), resemble predicted movement time value with the recorded movement time (MT) corresponding to the same task difficulty (ID). Table I shows details recorded data with calculated mean movement time and predicted movement time for a single user. The calculated coefficient correlation for this individual user was $\mathrm{r}=0.915$, which shows a strong relationship among MT and ID.

3) Distribution of $D$ and W over ID: Table II represents the 9 conditions of distance and width considered in this study and the calculated D/W ratio and index of difficulty (ID). Values of variable $\mathrm{D}$ and $\mathrm{W}$ are carefully considered to reveal IDs in range of 1 to 7 . A weight of .50 is added with each calculated ID to scale it and then rounded by flooring to integer value.

Before considering the final value for $\mathrm{D}$ and $\mathrm{W}$, an excel file is created to calculate different range of IDs, where several combinations of D and $\mathrm{W}$ is tested. Table III below represent a set of test data used in the excel file where values of distance variable (D) is increased by factor 2 and values of width variable decreased by the factor $1 / 2$ for every $D$ values.

\section{B. Analysis and Discussion}

1) Hypothesis 1: Observation of Fig. 4 shows an upward slope thus shows that Movement Time (MT) is linear with Index of Difficulty (ID), meaning it takes more time to perform more difficult task.

2) Fact 1: To improve pointing performance (reduce movement time) to a graphical target the difficulty level (ID) of a task needs to be reduced.

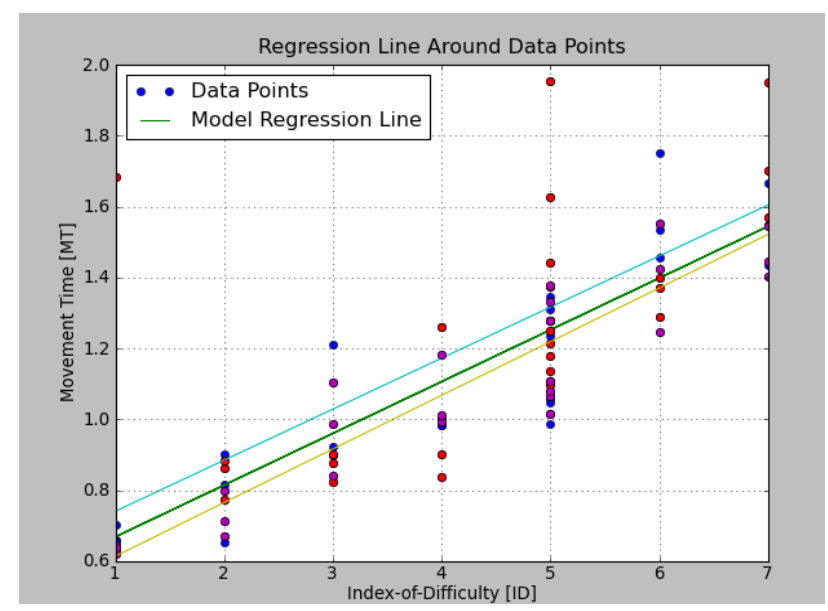

Fig. 4. ID vs. MT Graph
TABLE I. USER TRIAL DATA

\begin{tabular}{|l|l|l|c|c|c|}
\hline ID & $\begin{array}{c}\text { Recorded } \\
\text { Movement } \\
\text { Time }\end{array}$ & $\begin{array}{c}\text { Mean } \\
\text { Movement } \\
\text { Time }\end{array}$ & a & b & $\begin{array}{c}\text { Predicted } \\
\text { Movement } \\
\text { Time }\end{array}$ \\
\hline 1 & 0.703 & 0.673 & 0.46 & 0.15 & 0.614328 \\
\hline 1 & 0.659 & & 0.46 & 0.15 & 0.614328 \\
\hline 1 & 0.657 & & 0.46 & 0.15 & 0.614328 \\
\hline 2 & 0.816 & 0.789667 & 0.46 & 0.15 & 0.768306 \\
\hline 2 & 0.9 & & 0.46 & 0.15 & 0.768306 \\
\hline 2 & 0.653 & & 0.46 & 0.15 & 0.768306 \\
\hline 3 & 1.21 & 1.01 & 0.46 & 0.15 & 0.922285 \\
\hline 3 & 0.897 & & 0.46 & 0.15 & 0.922285 \\
\hline 3 & 0.923 & & 0.46 & 0.15 & 0.922285 \\
\hline 4 & 0.984 & 0.988667 & 0.46 & 0.15 & 1.076263 \\
\hline 4 & 1 & & 0.46 & 0.15 & 1.076263 \\
\hline 4 & 0.982 & & 0.46 & 0.15 & 1.076263 \\
\hline 5 & 1.109 & 1.134444 & 0.46 & 0.15 & 1.230242 \\
\hline 5 & 0.986 & & 0.46 & 0.15 & 1.230242 \\
\hline 5 & 1.058 & & 0.46 & 0.15 & 1.230242 \\
\hline 5 & 1.347 & & 0.46 & 0.15 & 1.230242 \\
\hline 5 & 1.237 & & 0.46 & 0.15 & 1.230242 \\
\hline 5 & 1.014 & & 0.46 & 0.15 & 1.230242 \\
\hline 5 & 1.312 & & 0.46 & 0.15 & 1.230242 \\
\hline 5 & 1.046 & & 0.46 & 0.15 & 1.230242 \\
\hline 5 & 1.101 & & 0.46 & 0.15 & 1.230242 \\
\hline 6 & 1.752 & 1.580667 & 0.46 & 0.15 & 1.384221 \\
\hline 6 & 1.456 & & 0.46 & 0.15 & 1.384221 \\
\hline 6 & 1.534 & & 0.46 & 0.15 & 1.384221 \\
\hline 7 & 1.548 & 1.549 & 0.46 & 0.15 & 1.538199 \\
\hline 7 & 1.434 & & 0.46 & 0.15 & 1.538199 \\
\hline 7 & 1.665 & & 0.46 & 0.15 & 1.538199 \\
\hline & & & & & \\
\hline & & & & & \\
\hline
\end{tabular}

TABLE II. CALCULATING ID

\begin{tabular}{|ll|l|ll|l|l|l|ll|}
\hline $\mathrm{D}$ & 20 & 40 & 60 & 20 & 40 & 60 & 20 & 40 & 60 \\
\hline $\mathrm{W}$ & 18 & 18 & 18 & 3 & 3 & 3 & 1 & 1 & 1 \\
\hline $\mathrm{DW}$ & 1.11 & 2.22 & 3.33 & 6.66 & 1333 & 20 & 20 & 40 & 60 \\
$\mathrm{I}$ (bit) & 1.15 & 2.15 & 2.73 & 3.73 & 4.73 & 5.32 & 5.32 & 6.32 & 690 \\
\hline $\begin{array}{l}\mathrm{ID} \\
(\mathrm{bit})+.50\end{array}$ & 1.65 & 2.65 & 3.13 & 4.13 & 5.13 & 5.82 & 5.82 & 6.82 & 7.40 \\
\hline $\operatorname{Int}(\mathrm{D})$ & 1 & 2 & 3 & 4 & 5 & 5 & 5 & 6 & 7 \\
\hline
\end{tabular}

TABLE III. D/W RATIO

\begin{tabular}{|l|l|l|ll|l|l|l|l|l|}
\hline $\mathrm{D}$ & 20 & 20 & 20 & 40 & 40 & 40 & 80 & 80 & 80 \\
\hline $\mathrm{W}$ & 18 & 9 & 4.5 & 18 & 9 & 4.5 & 18 & 9 & 4.5 \\
\hline $\mathrm{D} / \mathrm{W}$ & 1.11 & 2.22 & 4.44 & 2.22 & 4.44 & 8.88 & 3.33 & 6.66 & 13.33 \\
\hline $\begin{array}{l}\mathrm{ID} \\
\text { (bit) }\end{array}$ & 1.15 & 2.15 & 3.15 & 2.15 & 3.15 & 4.15 & 3.15 & 4.15 & 5.15 \\
\hline
\end{tabular}


3) Hypothesis 2: Table IV below is generated from Table I to compare the calculated mean movement time and predicted movement time.

When compared recorded movement time and predicted movement time with corresponding ID of table 4 it resembles quite same values for both. For smaller ID it differs more, which also a problem was shown in Fitts original experiment [14]. Another two different user trail data have been tested in the same manner with the excel file and it has been found that when there is strong correlation between MT and ID the predicted MT resembles similar values with recorded mean MT. Thus, it can be concluded that transmitting ID bits requires $\mathrm{a}+\mathrm{b}^{*}$ ID seconds in total, where $b$ is the slope and $a$ is the intercept calculated.

4) Fact 2: Since the predicting equation 2 can predict required movement time for a certain task, if any user takes more time to do that task the task can be identified by its ID and needs to be improved. Even times to be improved can be measured by subtracting the predicted movement time from the mean movement time.

5) Hypothesis 3: Table II shows that if the distance A to a target is increased by some factor $2(20,40$, and 80$)$ and the accuracy requirement is reduced ( $\mathrm{W}$ is increased) by the same factor $(\mathrm{W}=4.5,9$, and 18 increased by the factor 2$)$ the difficulty of the task (ID) stays same, meaning a target is twice as far and twice as large takes the same time to acquire.

6) Fact 3: When a task is identified as difficult one (requiring more time) the task can be improved either by resizing the target twice as large as its initial size or bring it twice as close as to the cursor current position.

Form above analysis and discussion on the hypotheses it is obvious that virtual pointing can be modelled using Fitts law and can result in performance very similar to physical pointing. When a physical pointing is not possible to improve, virtual pointing is very much possible to improve by reshaping and rearranging the target, thus "beating" Fitts law.

TABLE IV. MEANT MT AND PREDICTED MT

\begin{tabular}{|l|l|l|}
\hline ID & Recorded Mean MT & Predicted MT \\
\hline 1 & 0.673 & 0.614328 \\
\hline 2 & 0.789667 & 0.768306 \\
\hline 3 & 1.01 & 0.922285 \\
\hline 4 & 0.988667 & 1.076263 \\
\hline 5 & 1.134444 & 1.230242 \\
\hline 6 & 1.580667 & 1.384221 \\
\hline 7 & 1.549 & 1.538199 \\
\hline
\end{tabular}

\section{ADAPTIVE FITTS (MACHINE LEARNING)}

The aim of this part of the study is to affect changes in D and/or $\mathrm{W}$ in ways that result in shorter pointing times. Applicability of Fitts law has been demonstrated in this part of the study to show that virtual pointing using input device mouse can be improved by directly changing Fitts two

parameter (distance and width) in a systematic way. Changes of Fitts parameter does nothing more than change the size and position of on screen graphical elements (targets). Three facts have been taken in consideration form tested hypotheses in section 4 to derive advance form of computation for autotuning Fitts two parameters to produce dynamic user interface where the system intelligently applies changes to distance and size of a virtual target; thus, require machine learning.

\section{A. Predicting Mean Movement Time}

After collecting 540 trials data of 20 different subjects for a given set of ID (1 to 7) the mean movement time is calculated. The mean regression line is drawn on a scatter plot by regressing ID on MT (Figure 2). When slope $b^{\prime}$ (model mean slope) and intercept $a^{\prime}$ (model mean intercept) is worked out for the given set of data, for any known ID, the movement time is predicted and that is the mean movement time for that ID.

\section{B. User Movement Time}

When a new user or an existing user performs different set of tasks with the same set of IDs (1 to 7), user movement times (new set of movement times) are recorded. This new set of movement time is then compared with the mean movement time to identify user performance. When user movement time is higher than the mean movement time for any certain ID, user performance is poor and movement times need to be improved. The user movement time is known, IDs are known and slope $(b)$ and intercept $(a)$ is computed for this set of data.

\section{Achieving Predicted Movement Time}

If a user movement times need to be improved for any given task, the task difficulty (ID) needs to be worked out in a way so that the user can perform same task in shorter times. To achieve sorter movement time, it is obvious from hypothesis 1 that ID needs to be reduced.

\section{Reducing ID only by Reducing Distance}

This part of the study implemented a way of reducing ID without finding the amount of changes in slope and intercepts. When an existing user log in the system user performance is plotted back and compared with the mean regression line. User previous movement time is retrieved and compared with model mean movement time. Difficult task is represented graphically with previous settings where user took more time to point. After clicking the initial starting position difficult targets are brought closer to the mouse pointer by reducing distance by a fixed amount for each difficult task. Based on user new performance, slope and intercept are recalculated for that user and new set of ID is generated. It has been observed that user performance is improved. The new set of movement time and 
ID are generated to draw a new regression line and the regression line seems to be improved.

Fig. 5 below represents the model, where dark line is the mean regression line and, lighter colored line is the improved regression line of that user. Older IDs were integer number 1 to 7 and new improved IDs for corresponding older IDs are 1.0, $1.585,2.0,3.3219,4.585,5.5236$, and 6.0875

\section{E. Working out ID Correctly}

The ID is known, slope $(b)$, intercept $(a)$ and MT is known for a user. Also, the model (mean) slope $\left(b^{\prime}\right)$, model (mean) intercept $\left(a^{\prime}\right)$ and mean MT' is known. A new set of ID (improved ID) is now possible to work out to achieve the right movement time for each ID. The amount of ID need to be reduced can be calculated by finding the changes in slope and intercept and time differences between mean movement time and user movement time. An excel file is generated to test the data, table 6 show the results, using the data from Table 5. The computation and results are stated below-

$$
M T_{1}=a_{1}+b_{1} * I D_{i}
$$

In equation (4), $M T_{1}$ is mean movement time, $a_{1}$ is mean intercept, $b_{1}$ is mean slope, $i$ is the $n^{\text {th }}$ sequence of 1 to 7 and $I D$ is the index of difficulty.

$$
M T_{2}=a_{2}+b_{2} * I D_{i}
$$

In equation $5, M T_{2}$ is user (new) movement time, $a_{2}$ is user intercept, $b_{2}$ is user slope, $i$ is the $n^{\text {th }}$ sequence of (1 to 7) same $I D$ as in equation (i).

Since the ID is same and goal is to find the differences between mean movement time and user movement time and changes in slope and intercept in Fitts equation (equation 2) can be used as below

$$
\left(M T_{1}-M T_{2}\right)=\left(a_{1}-a_{2}\right)+\left(b_{1}-b_{2}\right) * I D_{i}
$$

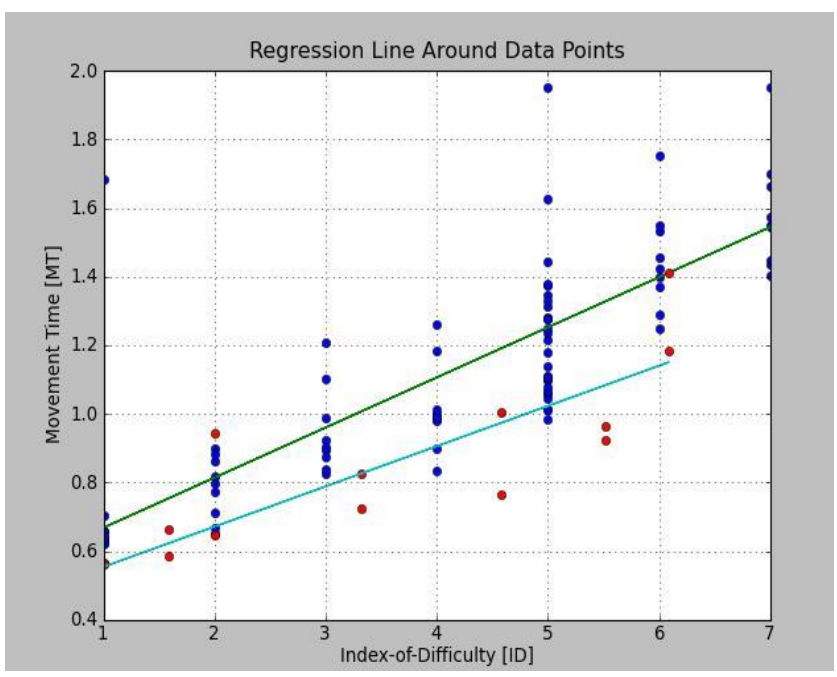

Fig. 5. Improved ID vs. MT
Intercept and slope is constant in Fitts equation thus changes of intercepts and slope with changes in ID is also constant. To compute the expected changes in movement time for one-unit changes in intercept and the unique effect of it on changes in movement time is decided by dividing changes in movement by changes in intercepts (which is constant), thus giving an equation-

$$
R_{1}=\frac{\left(M T_{1}-M T_{2}\right)}{\left(a_{1}-a_{2}\right)}
$$

where $R_{l}$ is ratio of the intercept changes in one-unit change of ID. To compute how changes in slope are distributed in changes of the ratio $R l$ needs to be divided by the constant changes in slope $\left(b_{1}-b_{2}\right)$, thus giving the equation-

$$
I_{i}=\frac{R_{1}}{\left(b_{1}-b_{2}\right)}
$$

Changes in $I D$ should be constant and it is verified by subtracting each next $I_{i+1}$ from $I_{i}$. As $I D$ is also a ratio of distance (A)/Width (W) the subtracted constant value $\left(I_{i+1}-I_{i}\right)$ is the amount of unit need to be increased in width (W) and/or decreased in distance (A) to reduce movement time. Table 7 below has been generated in excel using the above steps.

If the $\left(I_{i+1}-I_{i}\right)$ values gives negative value meaning user movement time is better than the model mean movement, although this can be verified at the very beginning by comparing mean movement with user movement time.

When $\left(I_{i+1}-I_{i}\right)$ values are either applied to distance or width values in Table 6 it generates a constant change in ID regarding previous ID. Table 6 gives the original value and then the effects of changes on it where distance is decreased by the constant $\left(I_{i+1}-I_{i}\right)$ value 8.397258 .

\section{CONCLUSION AND FUTURE WORK}

Throughout the study there was a process of understanding human motor performance by demonstrating Fitts law in virtual pointing task using mouse. A model has been developed to aid experiment in the interaction area of Human Computer Interaction (HCI). While $\mathrm{HCI}$ is the means of interaction between users and computers Graphical User Interface (GUI) makes it easier for user to interact with the system. This study shows that performance can be improved in point-and-click oriented task by slightly changing the design of the interface. The study described physical behavior of the user and interface to state that difficulty in computer task may arise due to lack of representation of graphical objects on the user interface.

This works offers working evidence in the domain of user interface showing that a dynamic target can provide better performance than static targets. Human performance and cursor movement data has been collected through a designed graphical measurement platform with the ordinary mouse. The study reveals that is relation between cursor movement and human motor performance. The work can be added to the previous studies that have tested Fitts law under different conditions and increased knowledge of its applicability.

The study would try to apply the same adaptation, tests, and experiments to touch screen based interface design. The smart 
devices offer several improvements such as adaptability and usability where applicability of Fitts law needs to be tested. Similar study on touch interfaces would provide recommendations for target size when interacting with fingers. Since powerful handheld devices are rapidly taking a place as peoples personal trusted device enabling these devices to be used in ever-increasing variety if task would require adaptivity.

\section{REFERENCES}

[1] John Mathers, 2015. DESIGN INTERVENTION. RSA Journal, Vol 161, No. 5561 (2015), pp. 24-29

[2] Chao Sun, Fazel Naghdy, and David Stirling, 2006. Application of MML to Motor Skills Acquisition. International Conference on Computational Intelligence for Modelling Control and Automation, and International Conference on Intelligent Agents, Web Technologies and Internet Commerce (CIMCA-IAWTIC'06), IEEE.

[3] Alan Dix, Janet Finlay, Gregory Abowd, and Russel Beale, 2004. Human-Computer Interaction. $3^{\text {rd }}$ ed.

[4] Andy Smith, 1997. Human-Computer Factors: A study of Users and Information Systems.

[5] B.C.M Smits-Engelman, G.P Van Galen and J. Duysens, 2002. The Breakdown of Fitts' Law in Rapid, Reciprocal Aiming Movements.

[6] Christopher Baber, 1997. Beyond the Desktop: Designing and Using Interaction Devices.

[7] CHI 2003: New Horizons. Short Talk: Fitts Law \& Text Input. Card, English, and Burr(1978)- 25 years later.

[8] CHI 2008 Proceedings-Fitt's Law Lives. Fitt's Throughput and SpeedAccuracy Trade-Off.

[9] David A. Rosenbaum, 1991. Human Motor Control.

[10] David H. McBurney and Virginia B. Collings, 1997. Introduction to Sensation/ Perception.

[11] D. Beamish, I Scott Mackenzie and Jianhong Wu, 2005. SpeedAccuracy Trade-Off in Planned Arm Movements with Delayed Feedback.

[12] George E. Stelmach, 1978. Information Processing in Motor Control and Learning.

[13] I. Scott MacKenzie, 1991. Fitts Law as a Performance Model in HumanComputer Interaction. [http://www.yorku.ca/mack/phd.html]

[14] I. Scott MacKenzie, 1995. Movement Time Prediction in HumanComputer Interface [Citeseerx.ist.psu.edu, 2003, (10.1.110.3904.pdf)]

[15] Michael John McGuffin, 2002. Fitts' Law and Expanding Targets: An Experimental Study, and Applications to User Interface Design.

[16] Paul M. Fitts and Michael I. Posner, 1979. Human Performance. Reprinted by Greenwood Press, Inc. USA

[17] Steven W. Keele, 1973. Attention and Human Performance.

[18] Thomas B. Sheridan and William R. Ferrell, 1981. Man-Machine Systems: Information, Control, and Decision Models of Human Performance.

[19] Rejean Plamondon and Adel M. Aimi, 1997. Speed/Accuracy TradeOffs in Target-Directed Movements, Behavioural and Brain Science20, p.279-49. [1997_hancock_verwey_bbs.pdf]

[20] Regan Lee Mandryk, 2000. Using the Finger for Interaction in Virtual Environments.

[21] Shumin Zhai, 2002. IBM Research Report: On the Validity of Throughput as a Characteristic of Computer Input. [www.almaden.ibm.com/u/zhai/papers/ZhaiIBMReporRJ10253.pdf]

[22] Shumin Zhai, Jing Kong and Xiangshi Ren, 2004. Speed-Accuracy Trade-Off Fitts' Law Tasks- On the Equivalency of Actual and Nominal Pointing

[www.almaden.ibm.com/cs/people/zhai/papers/Fits-SAT nalManuscript.pdf]

[23] Welford, A.T. (1968). Fundamentals of Skill. London: Methuen.

[24] Web Source, 2010. http://en.wikipedia.org/wiki/Mouse_(computing)
[25] Darko Brodić, and Alessia Amelio, 2016. Analysis of the HumanComputer Interaction on the Example of Image-Based CAPTCHA by Association Rule Mining. Symbiotic Interaction pp 38-51.

\section{Biography}

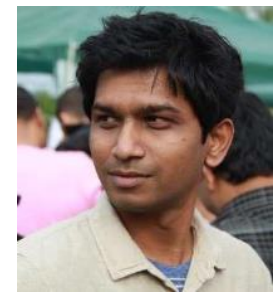

Sajib Hasan, was born on December 31, in a winter season in the year 1978. My birthplace is Pabna, Bangladesh. My father is a businessman in Pabna, where he won a Engineering Workshop. He has started his elementary study in Pabna Zilla School then moved to Rangpur Zilla School, where he have finished my schooling. Once he has finished his Higher Secondary from Rangpur Cantonment Public College, he went to the Philippines for higher study. Finishing undergraduate from AMA University of the Philippines, he came back in Bangladesh and started as a lecturer in Queens University, Dhaka, Bangladesh. Working there for two years and went to the United Kingdom for post-graduation. He has done his post-graduation from the University of Hertfordshire, U.K., and major in Artificial Intelligence and Robotics. He has finally returned to Bangladesh in the year of 2014. He has joined in the Computer Science and Engineering Department in the American International University-Bangladesh in the year 2014 and still in there. He started doing my $\mathrm{PhD}$ in Islamic University of Technology, Gazipur, Bangladesh. 